\title{
ANALYSE DE LA VARIABILITÉ MORPHOMÉTRIQUE ET MÉRISTIQUE DES POPULATIONS DE BARBUS CALLENSIS VALENCIENNES, 1842 (POISSONS, CYPRINIDÉS) DE TUNISIE.
}

\author{
M.M. KRAIEM
}

Laboratoire d'Hydroécologie, Département de Biologie, Faculté des Sciences de Tunis, Campus Universitaire, 1060 Tunis, TUNISIE.

\section{RÉSUMÉ}

Cette approche biométrique a été effectuée sur dix populations tunisiennes de Barbus callensis de provenances différentes. Elle a permis, grâce à l'analyse canonique discriminante, de mettre en évidence l'isolement écologique et génétique d'une population nord-ouest de toutes les autres populations du pays qui présentent une homogénéïté apparente. Tout en gardant leur pool génique original, les populations de $B$. callensis de Tunisie semblent devoir acquérir progressivement des caractères génétiques propres. Tunisie.

Mots-clés : Barbeau, Barbus callensis, variabilité, éco-génétique, populations,

\section{ANALYSIS OF MORPHOMETRIC AND MERISTIC VARIABILITY IN BARBUS CALLENSIS POPULATIONS (PISCES, CYPRINIDAE) FROM TUNISIA.}

\section{SUMMARY}

These biometrical investigations were performed on ten Tunisian populations of Barbus callensis collected in different streams, rivers and reservoirs all over the country. The statistical analysis (CAN-DISC) showed the ecological and genetical isolation of one northwestern population belonging to an Algerian catchment from the other Tunisian populations, who appeared more uniform. While preserving their original gene pool, most Tunisian strains seem to be acquiring original characters among NorthAfrican $B$. callensis.

Key-words : Barbel, Barbus callensis, variability, eco-genetic, populations, Tunisia.

\section{INTRODUCTION}

En Tunisie, le genre Barbus est essentiellement représenté par l'espèce $B$. callensis qui présente une large répartition biogéographique. Celle-ci s'étend sur tout le nord et le centre-ouest du pays (KRAIEM, 1983), occupant ainsi divers milieux. 
Dans le cas des études des poissons des eaux courantes, la notion de population est parfois escamotée par la complexité et la difficulté d'approche de ces milieux. Ainsi, une structure géographique linéaire ramifiée telle que celle du domaine fluvial constitue écologiquement un élément habituel de différenciation populationnelle (LOUDENSLAGER et GALL, 1980).

La présence de plusieurs populations, que ce soit dans un même cours d'eau, dans des cours d'eau différents, ou dans des bassins différents, suppose un certain isolement reproductif. Cet isolement reproductif peut se traduire par une évolution divergente entre populations, c'est-à-dire une modification du pool génique. L'ensemble du génome détermine les caractéristiques morphologiques et physiologiques des individus, c'est-à-dire leur capacité de survie. La sélection naturelle tend à produire des populations adaptées aux caractéristiques du milieu aus sein duquel elles évoluent.

Dans la pratique, les études en biologie des populations se basent, le plus souvent, sur l'analyse du génome et l'examen de l'aspect extérieur des individus. Les approches les plus utilisées de nos jours sont, de loin, la biométrie et l'analyse biochimique des protéines. La présente étude sera basée sur la première approche et aura pour objectif d'estimer l'homogénéité de $B$. callensis en Tunisie, en rapport avec les caractéristiques écologiques des divers bassins hydrographiques du pays qu'il occupe.

\section{MATÉRIEL ET MÉTHODES}

\section{Etude des milieux prospectés}

Les différents échantillons de barbeaux proviennent des localités mentionnées sur la carte (Fig. 1).

Le choix de ces lieux de prélèvement repose sur la particularité de leurs bassins versants. II s'agit :

— du bassin du Nord-Ouest pour l'oued Ennour, qui est un affluent d'altitude $(450 \mathrm{~m})$ de l'oued El Kébir qui coule en Algérie ; et l'oued Ghézala, un affluent secondaire du fleuve Mejerda,

- du bassin de l'Ichkeul, pour les oueds Sejnane et Joumine situés dans la plaine de Mateur,

— du bassin de la Mejerda, pour l'o. Mejerda à Sidi Salem et ses affluents, l'o. Béja et l'o. Siliana,

— du bassin du Nord-Est pour les oueds Abid et Miliane,

- du bassin du Centre pour l'oued Nabhana, situé en zone semi-aride au nord de Kairouan.

Les caractéristiques morphodynamiques et physicochimiques qui nous semblent fondamentales ont été relevées et regroupées dans les tableaux I et II.

\section{Traitement des échantillons de Barbeaux}

Les poissons ont été récoltés pour la plupart par pêche électrique, mais ceux provenant des milieux à forte conductivité ont été capturés par filets maillants et artisanaux. Ils ont été conservés sur plẩce dans une solution formolée à $4 \%$. Les échantillons ont été prélevés entre 1986 et 1991.

L'étude a porté sur 220 barbeaux de taille comprise entre 5 et $30 \mathrm{~cm}$ (Lt) provenant de 10 milieux appartenant aux différents bassins situés dans l'aire de répartition de cette espèce : 


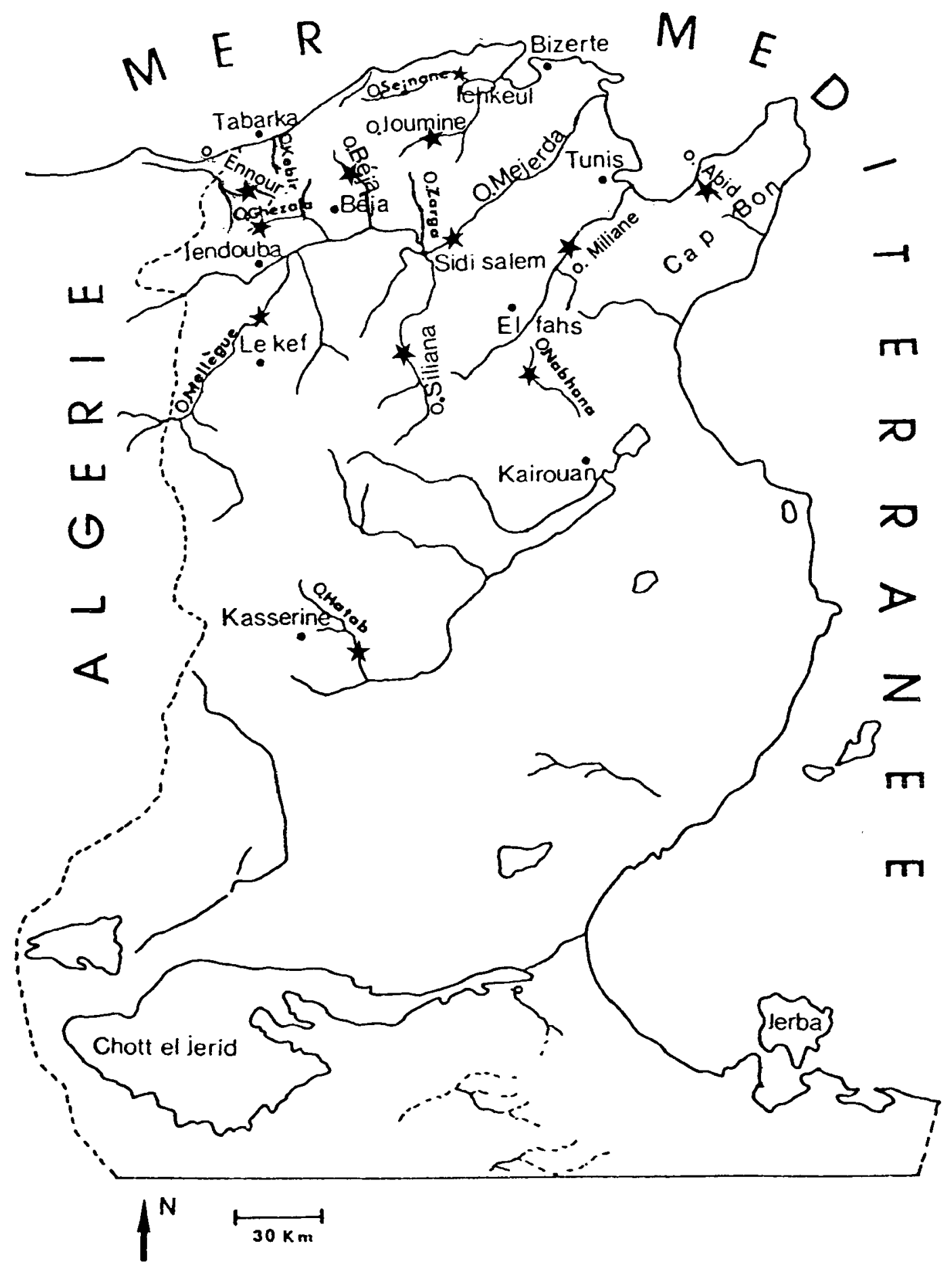

Figure 1 : Cours d'eau prospectés et localisation des stations d'étude.

Figure 1 : Prospected streams and location of study sites. 


\begin{tabular}{|c|c|c|c|}
\hline - Oued Ennour & $E$ & \multicolumn{2}{|c|}{ (14 spécimens ) } \\
\hline - 0. Ghézala : & $\mathrm{G}$ & $(24$ & « \\
\hline - O. Béja : & $\mathrm{B}$ & $(19$ & « \\
\hline - O. Mejerda à Sidi Salem: & $M$ & $(17$ & « \\
\hline — O. Siliana & $S$ & $(24$ & “ \\
\hline - O. Joumine: & $J$ & $(24$ & « \\
\hline - O. Sejnane & C & $(38$ & « \\
\hline - O. Abid: & $A$ & $(14$ & « \\
\hline — O. Miliane : & $L$ & $(25$ & « \\
\hline - O. Nabhana: & $N$ & $(21$ & $«$ \\
\hline
\end{tabular}

La description des poissons porte sur deux types de caractères :

- des caractères numériques (méristiques) qui correspondent au dénombrement de diverses pièces anatomiques (écailles, rayons des nageoires, branchiospines, etc...),

- et des caractères morphométriques qui correspondent à des mensurations de certains éléments du corps.

Les caractères méristiques correspondent à des variables discrètes qui fournissent des données objectives du fait qu'elles sont indépendantes de la taille du sujet examiné. Par contre, les caractères morphométriques correspondent plutôt à des variables continues corrélées avec la taille du sujet et présentent un caractère plus subjectif dans la mesure où la façon de mesurer est plus sujette à variation de la part de l'opérateur.

\section{Analyse biométrique}

Cette analyse a été effectuée sur les Barbeaux appartenant aux 10 populations citées plus haut. Chacune d'elles est décrite par 14 caractères. Les caractères retenus représentent 5 méristiques et 9 morphométriques ; ces derniers sont traduits sous forme de rapports, ils sont ainsi réduits à 7 . L'étude biométrique générale portera alors sur 12 variables (Tableau III). Nous avons donc, pour chaque caractère, 220 observations.

Tableau III : Codes des variables retenues pour l'analyse canonique discriminante.

Table III : Variables codes used for the canonical discriminant analysis.

A - Variables méristiques

Codes

1) Nombre total de vertèbres $\quad$ V1

2) Nombre de dents sur le $4^{\ominus}$ rayon osseux de la nageoire dorsale V2

3) Nombre de branchiospines sur le coté supérieur du $1^{\mathrm{er}}$ arc branchial gauche V3

4) Nombre de branchiospines sur le côté inférieur du $1^{\text {er }}$ arc branchial gauche V4

5) Nombre d'écailles sur la ligne latérale

$\vee 5$

B - Variables morphométriques transformées

6).... V7/V6 longueur de la tête/longueur standard $\quad$ T1

7)......V8N7 diamètre de l'œil/longueur de la tête $\quad$ T2

8)......V9/V6 longueur de la nageoire pectorale/longueur standard

9)......V10/N6 distance entre les nageoires ventrales et l'anale/longueur standard T4

$10) \ldots . . . V 11 / \mathrm{V} 6$ distance pré-dorsale/longueur standard $\quad$ T5

11)......V12/V6 distance pré-ventrale/longueur standard $\quad$ T6

12)......V14/N13 longueur de la partie denticulée du $4^{\text {e }}$ rayon osseux de la nageoire $T 7$ dorsale/longueur totale du même rayon 
Pour le traitement statistique des données, nous avons fait appel à «'Analyse Canonique Discriminante" (YOCCOZ, 1988 ; ZAIEM, 1988). Cette analyse multivariée permet de mettre en évidence la ou les dimensions selon lesquelles les groupes se distinguent le mieux. Plusieurs auteurs (RAO, 1953 et 1973 ; ROUVIER, 1966 ; HEBRANT, $1974 \ldots)$ ont montré que l'on peut établir des combinaisons linéaires discriminantes de plusieurs observations qui rendent le mieux compte de la dispersion de ces observations entre deux classes, deux espèces taxonomiques ou deux populations.

L'analyse a été effectuée selon un programme S.A.S. (Statistical Analysis System), qui nous a été aimablement fourni par le Centre de Calcul EL KHAWAREZMI de TUNIS ; nous avons utilisé la procédure CAN-DISC.

\section{RÉSULTATS}

\section{Analyse écologique}

Les eaux de surface en Tunisie sont essentiellement concentrées dans le Nord, correspondant à $80 \%$ des potentialités hydrologiques du pays. L'examen des données morphodynamiques (tableau I) des différentes stations prospectées nous permet de distinguer 3 catégories d'oueds:

- les oueds d'altitude, de faibles dimensions et à cours plutôt rapide plus ou moins interrompu en période d'étiage (oueds Ennour et Ghézala),

- les oueds de plaine, de grandes dimensions et pérennes (oueds Mejerda, Miliane, Joumine et Nabhana,

- les oueds de plaine, de dimensions moyennes et à cours plus ou moins interrompu en période d'étiage (oueds Sejnane, Béja, Siliana et Abid).

Tableau I : Caractères morphodynamiques des milieux prospectés ( $\mathbf{R}:$ rochers ; $\mathbf{P}$ : pierres ; $\mathbf{G}$ : graviers ; $\mathbf{S}$ : sable).

Table I : Morphodynamic characteristics of the prospected biotopes (R : rocks ; $P$ : stones ; G : gravel ; $\mathbf{S}$ : sand).

\begin{tabular}{|c|c|c|c|c|c|c|c|}
\hline Oueds & Affluence & Altitude (m) & Pente $(\%)$ & Largeur (m) & Substrat & $\begin{array}{l}\text { Vitesse du } \\
\text { courant }\end{array}$ & $\begin{array}{c}\text { "Cours } \\
\text { accidenté» }\end{array}$ \\
\hline Ennour & $\begin{array}{l}\text { O. El Kébir } \\
\text { Algérie }\end{array}$ & 410 & 4,17 & $4 \cdot 2,5$ & $R+P$ & Rapide & Oui \\
\hline Ghézala & $\begin{array}{l}\text { O. Bouhertma } \\
\text { et } O \text {. Mejerda }\end{array}$ & 250 & 0,43 & $5-3,5$ & $G+P+S$ & Rapide & Non \\
\hline Béja & O. Mejerda & 155 & 0,16 & $5-3,0$ & $P+S$ & $\begin{array}{c}\text { Rapide à } \\
\text { moyen }\end{array}$ & à l'étiage \\
\hline $\begin{array}{l}\text { Retenue } \\
\text { Sidi Salem }\end{array}$ & O. Mejerda & 145 & 0,35 & S : 4300 ha & $R+P+S+V$ & $\begin{array}{l}\text { Moyen à } \\
\text { calme }\end{array}$ & Non \\
\hline Sejnane & O. Sejnane & 10 & 0,14 & $6-3,5$ & $P+G+S$ & Moyen & Oui \\
\hline Joumine & O. Joumine & 15 & 0,27 & $6-4,0$ & $P+G+S$ & $\begin{array}{l}\text { Moyen à } \\
\text { rapide }\end{array}$ & Non \\
\hline Abid & O. Abid & 10 & 0,28 & $5-3,0$ & $P+S$ & Moyen & $+/-$ \\
\hline Miliane & O. Miliane & 170 & 0,22 & $6-4,0$ & $R+P+S$ & $\begin{array}{l}\text { Moyen à } \\
\text { rapide }\end{array}$ & Non \\
\hline Siliana & O. Mejerda & 420 & 1,50 & $6-4,0$ & $P+S$ & $\begin{array}{l}\text { Moyen à } \\
\text { rapide }\end{array}$ & Non \\
\hline Nabhana & O. Nabhana & 90 & 0,31 & $6-4,0$ & $P+G+S$ & $\begin{array}{l}\text { Moyen à } \\
\text { rapide }\end{array}$ & $+/-$ \\
\hline
\end{tabular}


D'autre part, l'analyse des données physicochimiques relevées sur 3 années consécutives : 1988 - 89 - 90 (tableau II) montre que les eaux des différents milieux ne se différencient nettement que par les variations de la salinité. En effet, la composition en sels est généralement stable ou varie peu ; sur l'ensemble des stations, la salinité $\mathbf{S}$ mesurée aux quatre saisons (tableau II) permet de définir :

- une région nord-ouest à faible salinité : oueds Ennour, Ghézala et Béja, avec $0,1<\mathrm{S}<0,4 \mathrm{~g} / \mathrm{l}$ en hiver et $0,2<\mathrm{S}<0,7 \mathrm{~g} / \mathrm{l}$ en été,

- une région côtière nord, à très forte amplitude de variation : oueds Sejnane, Joumine et Abid avec respectivement 0,$4 ; 0,4$ et $0,3 \mathrm{~g} / \mathrm{l}$ en hiver et 2,$5 ; 1,2$ et $1,1 \mathrm{~g} / \mathrm{l}$ en été,

- une région centre à forte salinité : elle comprend tous les autres cours d'eau analysés. La salinité en hiver ne descend que rarement au dessous de $1 \mathrm{~g} / \mathrm{l}$ et dépasse toujours cette valeur en été, avec un maximum de $3 \mathrm{~g} / \mathrm{l}$ mesuré dans l'oued Miliane.

Ce gradient de salinité nord-sud a déjà été observé (KALLEL, 1984) avec des valeurs inférieures à $1,5 \mathrm{~g} / \mathrm{l}$ au nord, $1,6 \mathrm{~g} / \mathrm{l}$ à Sidi Salem et 1,8 $\mathrm{g} / \mathrm{l}$ dans l'oued Nabhana, la plus au sud des stations étudiées.

Les autres mesures de sels dissous confirment la principale partition du pays en régions nord-ouest et centre-sud avec une forte augmentation des concentrations en $\mathrm{SO}_{4}{ }^{--}$ $\mathrm{Cl}^{-}, \mathrm{NO}_{3}{ }^{-}$et $\mathrm{CaCO}_{3}$ vers le centre-sud (tableau II).

Tableau II : Physicochimie des eaux des différents milieux prospectés (valeurs extrêmes des relevés effectués, en cours de journée, sur 3 années successives : 1988-89-90).

Table II : Water physicochemical parameters of the various prospected biotopes (extreme values of measurements carried out, during the day, along 3 successive years : 1988-89-90).

\begin{tabular}{|c|c|c|c|c|c|c|c|c|c|c|}
\hline Oueds & $\begin{array}{l}t^{\circ} \mathrm{C} \\
\text { (eau) }\end{array}$ & $\begin{array}{c}\mathrm{O}_{2} \\
(\mathrm{mg} / \mathrm{l})\end{array}$ & $\begin{array}{c}\text { Salinité } \\
(\mathrm{g} / \mathrm{l})\end{array}$ & $\begin{array}{l}\text { Cond. }\left(20^{\circ} \mathrm{C}\right) \\
\left(\mu \mathrm{s} / \mathrm{cm}^{2}\right)\end{array}$ & $\mathrm{pH}$ & $\begin{array}{l}\mathrm{SO}_{4}^{-} \\
(\mathrm{mg} / \mathrm{l})\end{array}$ & $\underset{(\mathrm{mg} / \mathrm{l})}{\mathrm{Cl}^{-}}$ & $\begin{array}{l}\mathrm{NO}_{3}^{-} \\
(\mathrm{mg} / \mathrm{l})\end{array}$ & $\begin{array}{l}\text { Dureté totale } \\
\left(\mathrm{mg} \mathrm{CaCO}_{3} /\right)\end{array}$ & $\begin{array}{c}\text { Alcalinité } \\
\left(\mathrm{mg} \mathrm{HCO}_{3} /\right)\end{array}$ \\
\hline 0. Ennour & $5,1-27,2$ & $7,8-10,5$ & $0,1-0,2$ & $230-420$ & $7,8-8,3$ & $30-81$ & $30-50$ & $0,05-0,25$ & $100-130$ & $60-200$ \\
\hline 0. Ghézala & $5.4-27,8$ & $7,6-9,8$ & $0,1-0,6$ & $354-1210$ & $7,8-8,4$ & $80-195$ & $30 \cdot 150$ & $0,27-1,15$ & $150-215$ & 250 \\
\hline 0. Béja & $5,8-28,7$ & $7,2-9,7$ & $0,2-0,7$ & $567-1400$ & $8,0-8,3$ & $85-180$ & $80-180$ & $1,05-9,90$ & 120 & 330 \\
\hline Sidi Salem & $6,2-28,4$ & $7,6-9,5$ & $1,6-2,3$ & $1760-2540$ & $8,1-8,4$ & $500-610$ & $400-520$ & $0,94-1,80$ & $600-700$ & $150-180$ \\
\hline 0. Joumine & $6,4-28,7$ & $7,8-10,7$ & $0,4-1,2$ & $450-1550$ & $8,2-8,5$ & $60-170$ & $60-100$ & $2,50-8,20$ & $360-430$ & $180-200$ \\
\hline 0. Sejnane & $7,2-29,1$ & $7,1-11,9$ & $0,4-2,5$ & $546-3900$ & $8,2-8,7$ & $45-90$ & $160-430$ & $1,25-3,70$ & $450-700$ & $130-250$ \\
\hline 0. Abid & $7,1-29,3$ & $6,3-9,1$ & $0,3-1,4$ & $520-2215$ & $7,8-8,2$ & $130-170$ & $170-570$ & $0,82-1,35$ & $300-370$ & $100-380$ \\
\hline 0. Miliane & $7,4-29,6$ & $7,2-11,7$ & $2,0-3,1$ & $2100-6400$ & $8,2-8,6$ & $900-1500$ & $450-1960$ & $1,95-4,65$ & $600-1300$ & $170-360$ \\
\hline o. Siliana & $7,8-30,1$ & $7,8-11,5$ & $0,8-1,3$ & $1100-1700$ & $7,8-8,2$ & $250-460$ & $50-215$ & $4,87-8,92$ & $500-600$ & $160-215$ \\
\hline 0. Nabhana & $8,2-31,2$ & $7,8-9,8$ & $0,8-1,6$ & $2100-2900$ & $7,6 \cdot 8,7$ & $400-610$ & $200-570$ & $0,28-1,54$ & $380-930$ & $110-230$ \\
\hline
\end{tabular}


Les autres facteurs tels que l'oxygène dissous, le $\mathrm{pH}$ ou l'alcalinité $\left(\mathrm{HCO}_{3}\right)$ et la dureté ne présentent pas de contraste nord/sud, à l'exception de la conductivité qui est essentiellement fonction de la salinité et de la température qui reflète un certain réchauffement des eaux du nord vers le sud.

\section{Analyse des différentes populations de $B$. callensis}

\section{Structures canoniques}

Les variables canoniques sont choisies de façon à assurer un rapport maximum des différences entre groupes par comparaison avec les différences existant à l'intérieur des groupes. On détermine ainsi les coefficients canoniques standardisés des variables qui permettent de définir les composantes canoniques, ainsi que la quantité d'absorption de la variabilité par ces composantes.

\section{a) Définition des axes canoniques}

Le tableau IV représente les structures canoniques qui constituent les corrélations des caractères initiaux avec les axes canoniques.

La première composante canonique (Can. 1) est fortement corrélée avec $V_{3}$ : le nombre de branchiospines sur le côté supérieur du $1^{\text {er }}$ arc branchial gauche. D'autres variables pourraient être corrélées à un degré moindre avec cet axe; il s'agit de $V_{5}$ : nombre d'écailles sur la ligne latérale et $V_{2}$ : nombre de dents sur le $4^{e}$ rayon osseux de la nageoire dorsale.

La deuxième composante canonique (Can.2) est définie essentiellement par $V_{4}$ : nombre de branchiospines sur le côté inférieur du $1^{\text {er }}$ arc branchial gauche.

Tableau IV : Structures canoniques (a) et coefficients canoniques standardisés (b) des populations de $B$. callensis étudiées.

Table IV : Canonical structures (a) and standardized canonical coefficients (b) of the studied $B$. callensis populations.

\begin{tabular}{rlrlllllllllll}
\hline $\begin{array}{c}\text { Caractères } \\
\text { Axes }\end{array}$ & V1 & V2 & V3 & V4 & V5 & T1 & T2 & T3 & T4 & T5 & T6 & T7 \\
\hline Can 1 (a) & $-0,416$ & 0,169 & 0,926 & 0,458 & $-0,870$ & $-0,610$ & $-0,171$ & $-0,041$ & $-0,127$ & $-0,441$ & $-0,655$ & 0,723 \\
& (b) & $-0,177$ & $-0,578$ & 0,979 & 0,530 & $-0,690$ & $-0,127$ & 0,087 & 0,206 & $-0,118$ & $-0,049$ & $-0,113$ & 0,329 \\
\hline Can 2 & (a) & 0,163 & 0,824 & $-0,048$ & 0,842 & 0,179 & $-0,247$ & $-0,548$ & 0,699 & 0,818 & $-0,184$ & $-0,118$ & 0,332 \\
& (b) & 0,119 & 0,545 & $-0,574$ & 0,980 & 0,090 & 0,062 & $-0,024$ & 0,193 & 0,301 & $-0,229$ & 0,266 & 0,326 \\
\end{tabular}

b) Absorption de la variabilité des caractères par les axes canoniques

D'après le Tableau $V$, l'axe Can.1 défini par $\left(V_{3}, V_{5}\right.$ et $\left.V_{2}\right)$ absorbe $35,7 \%$ de la variabilité ; l'axe Can.2 défini par $\left(\mathrm{V}_{4}\right)$ absorbe $28,4 \%$ de la variabilité. Ainsi, à elles deux, les composantes Can. 1 et Can. 2 absorbent $64,1 \%$, soit environ les $2 / 3$ de la variabilité. Nous allons donc nous limiter à ces deux composantes pour établir les projections des populations sur les plans qu'elles engendrent.

La construction des polygones délimitant les différentes populations a permis de mettre en évidence deux groupes de populations (Fig. 2).

Un premier groupe bien isolé des autres est constitué par la population de l'oued Ennour, caractérisé par: 
Bull. Fr. Pêche Piscic. (1994) $334: 201-212-208-$

Tableau V : Absorption de la variabilité par les axes canoniques.

Table $\mathrm{V}$ : Variability absorption by the canonical axes.

\begin{tabular}{ccc}
\hline Axes & Can. 1 & Can. 2 \\
\hline Corrélation canonique & 0,790 & 0,755 \\
Erreur standard & & \\
approximative & 0,025 & 0,029 \\
Valeur propre & 1,669 & 1,328 \\
Pourcentage d'inertie & 35,70 & 28,40 \\
Inertie cumulée & 35,70 & 64,10 \\
\hline
\end{tabular}

- un nombre relativement important de denticulations (26 en moyenne) sur le dernier rayon osseux de la nageoire dorsale,

- un nombre relativement important de branchiospines (4 sur le côté supérieur et 8 sur le côté inférieur du $1^{\text {er }}$ arc branchial gauche),

— une tête plus petite, et un cil plus petit.

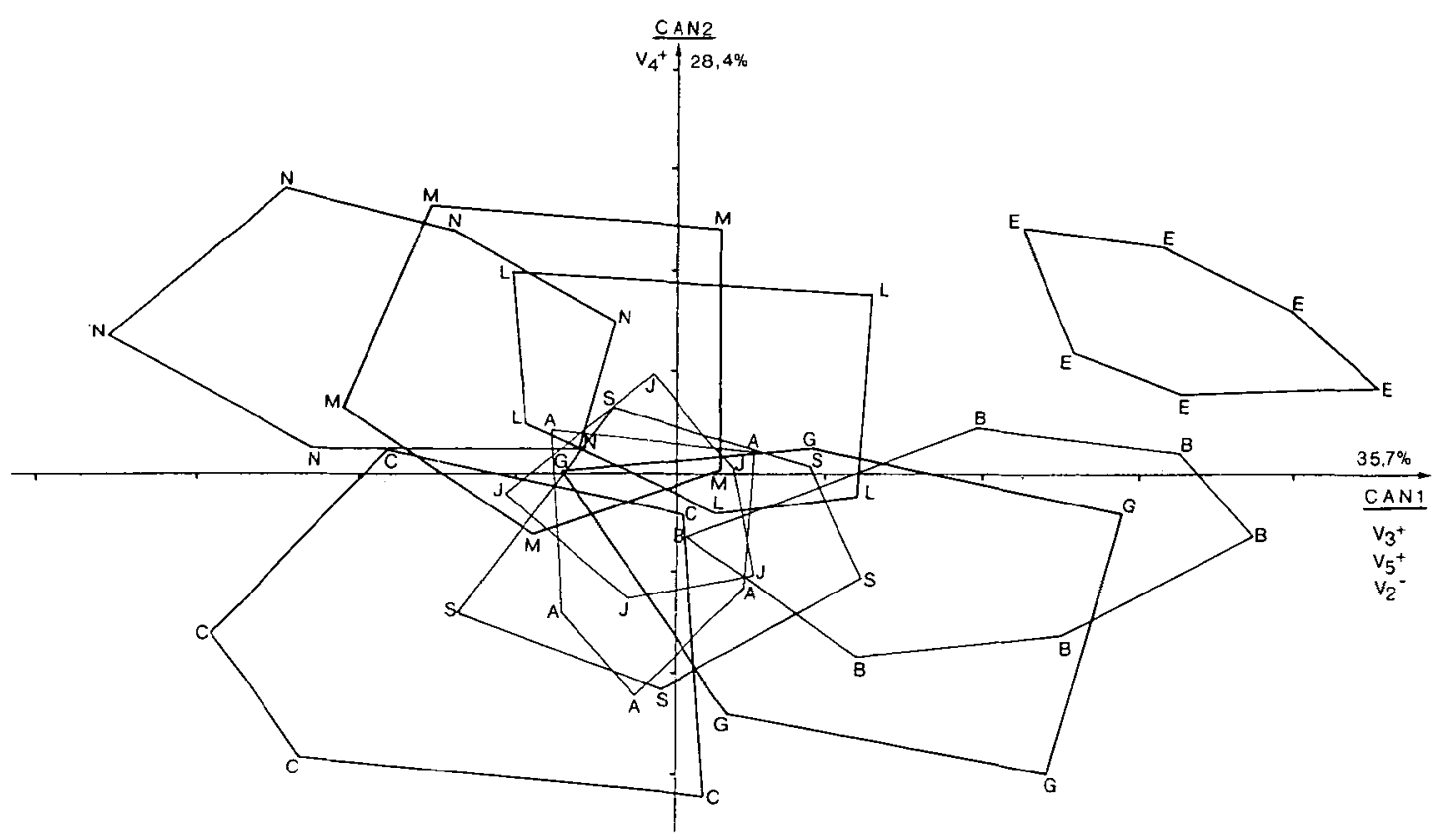

Figure 2 : Représentation graphique de l'analyse canonique discriminante relative aux diverses populations de $B$. callensis étudiées (légende dans le texte).

Figure 2 : Graphic representation of the canonical discriminant analysis relative to the various studied populations of $B$. callensis (legend in the text). 
Un deuxième groupe caractérisé par :

- un nombre plus faible de denticulations sur le dernier rayon osseux de la nageoire dorsale (la moyenne varie entre 17 et 23 ),

- un nombre de branchiospines plus faible (entre 2 et 3 pour le côté supérieur, et entre 5 et 7 pour le côté inférieur du $1^{\text {er }}$ arc branchial gauche),

— une tête et un œil relativement plus développés,

semble être constitué de deux sous-groupes. Le premier rassemble les populations des oueds Abid, Siliana, Joumine, Miliane, Mejerda, Nabhana et Sejnane ; et le second regroupe les populations des oueds Béja et Ghézala, ces deux populations situées du côté positif de l'axe Can.1 semblent constituer un groupe charnière entre le premier sous-groupe et la population d'Ennour. Elles se rapprochent de cette dernière par les caractères suivants :

- un nombre d'écailles de $42 \pm 1$, alors que chez les autres populations il est de $43 \pm 1$,

- des denticulations nombreuses qui occupent une distance relativement courte sur le dernier rayon osseux de la nageoire dorsale.

\section{Distances de Mahalanobis $\left(\mathrm{D}^{2}\right)$}

Ces distances permettent de situer plusieurs populations dans un espace à $p$ dimensions ( $p$ étant le nombre de variables ou de caractères) et déterminent dans quelle mesure ces populations peuvent être distinguées les unes des autres (DAGNELIE, 1982).

Les distances de Mahalanobis entre les différentes populations de $B$. callensis sont portées sur le tableau VI. Ces distances sont estimées à travers l'ensemble des caractères ; leur comparaison a été réalisée à l'aide du test $F$.

Tableau VI : Distances de Mahalanobis entre les différentes populations de B. callensis étudiées (légende dans le texte).

Table VI : Mahalanobis distances between the various $B$. callensis studied populations (legend in the text).

\begin{tabular}{|c|c|c|c|c|c|c|c|c|c|c|}
\hline Populations & $A$ & B & $C$ & $\mathrm{G}$ & $E$ & $\mathrm{~J}$ & $\mathrm{~L}$ & $M$ & $\mathrm{~N}$ & $S$ \\
\hline A & 0 & 5,751 & 4,642 & 5,999 & 21,662 & 6,926 & 6,797 & 9,065 & 8,446 & 7,008 \\
\hline B & & 0 & 9,349 & 4,641 & 14,488 & 6,734 & 9,001 & 13,809 & 17,911 & 6,867 \\
\hline C & & & 0 & 8,123 & 28,585 & 4,834 & 7,629 & 10,601 & 9,708 & 8,053 \\
\hline $\mathrm{G}$ & & & & 0 & 14,401 & 7,173 & 9,679 & 15,840 & 15,662 & 10,885 \\
\hline$E$ & & & & & 0 & 17,931 & 14,369 & 18,264 & 27,950 & 22,666 \\
\hline$J$ & & & & & & 0 & 3,887 & 5,442 & 8,962 & 8,521 \\
\hline$L$ & & & & & & & 0 & 4,673 & 4,420 & 9,079 \\
\hline$M$ & & & & & & & & 0 & 5,253 & 10,116 \\
\hline$N$ & & & & & & & & & 0 & 13,324 \\
\hline S & & & & & & & & & & 0 \\
\hline
\end{tabular}


Ces distances permettent par la suite d'établir un dendrogramme qui classe les différentes populations selon leurs degrés de proximité (SNEATH et SOKAL, 1973) :

$$
I p=\frac{1}{D^{2}} \times 100
$$

Nous constatons que les plus grandes distances sont celles qui séparent la population d'Ennour des autres populations. Par ailleurs, les distances les plus importantes se situent entre cette dernière et celle du Sejnane $(28,58)$, celle du Nabhana $(27,95)$ et celle du Siliana $(22,66)$. Signalons, en outre, que toutes les distances séparant les différentes populations sont significatives.

L'établissement du dendrogramme des indices de proximité des populations (Fig.3) déduits des distances de Mahalanobis permet de distinguer plus nettement les groupes de populations. Ainsi nous distinguons, d'après l'arbre constitué, la population bien isolée de l'oued Ennour, oued d'altitude, de faibles dimensions, et à cours interrompu en période d'étiage, qui présente des eaux très douces, fraîches, claires et propres, et le groupe formé par les autres populations qu'on peut diviser en deux sous-groupes. Le premier renferme les populations des oueds Joumine, Miliane, Mejerda et Nabhana, oueds de plaine pérennes et de grandes dimensions, caractérisés (à l'exception de l'o. Joumine) par des eaux plus salées, plus réchauffées, plus turbides et moins propres. Le deuxième regroupe les populations des oueds Abid, Sejnane; Béja, Ghézala et Siliana ; ces oueds de plaine mais de dimensions moyennes se caractérisent par des eaux de qualité physico-chimique intermédiaire et un cours plus ou moins permanent. Ceci explique la position centrale de ces populations par rapport à celles d'Ennour d'une part, et de Joumine, Miliane, Mejerda et Nabhana, d'autre part.



Figure 3 : Dendrogramme des indices de proximité des différentes populations de $B$. callensis étudiées (légende dans le texte).

Figure 3 : Proximity index dendrogram of the various studied populations of $B$. callensis (legend in the text). 


\section{DISCUSSION ET CONCLUSION}

Cette étude biométrique nous a permis, grâce à l'utilisation de l'analyse canonique discriminante,

- d'une part, de limiter le nombre de caractères étudiés en mettant en évidence ceux qui discriminent le plus les populations. Ces caractères sont essentiellement le nombre de branchiospines du $1^{\text {er }}$ arc branchial, le nombre d'écailles sur la ligne latérale, le nombre de dents sur le $4^{e}$ rayon osseux de la nageoire dorsale et le rapport du diamètre de l'oeil à la longueur de la tête ;

- d'autre part, de classer les différentes populations selon les deux premières composantes canoniques et de les comparer.

L'apparition de deux ensembles bien individualisés a permis de mettre en évidence l'existence d'une population "originale» au nord-ouest (celle d'Ennour). Celle-ci, appartenant à un bassin versant indépendant situé au nord-est de l'Algérie, se trouve géographiquement isolée, ce qui laisse supposer des échanges géniques faibles voire absents entre cette population et toutes les autres.

Cependant, certaines des autres populations, en l'occurrence celles de Khroumirie (Béja et Ghézala) géographiquement proches de celle d'Ennour, semblent présenter avec celle-ci des affinités morphologiques qui tendent à les rapprocher. Mais, d'un autre côté, la grande similitude que présentent les deux populations des oueds Béja et Ghézala avec les autres populations de l'intérieur du pays les retient dans cet ensemble. L'appartenance de ces populations à des bassins versants qui communiquent pourrait engendrer un brassage génique qui expliquerait ces similitudes.

D'autre part, bien qu'elle soit géographiquement plus éloignée de celle d'Ennour et occupant un milieu écologiquement différent, la population du Miliane paraît phénotypiquement aussi proche d'elle que les populations de Béja et de Ghézala. Nous retrouvons ici la distorsion, constatée lors de l'approche biochimique que nous avons déjà entreprise (BERREBI et al., en cours), entre les caractéristiques écologiques du milieu et les caractéristiques génétiques des barbeaux qui les peuplent car l'oued Miliane, dont la population est génétiquement rattachée au groupe d'Ennour et du Nord-Ouest (Fig. 2), est écologiquement nettement du groupe Centre. De plus, l'o. Joumine, qui présente des caractères écologiques typiques des oueds du Nord, voit sa population classée génétiquement dans le groupe du Centre (Fig. 3).

Malgré l'homogénéité apparente du grand ensemble, nous remarquons certaines différences entre les populations de $B$. callensis auxquelles s'ajoutent les distorsions entre l'écologie et la génétique de ces populations. Ce qui nous laisse supposer que cette différenciation aurait une origine paléo-historique liée aux difficultés de colonisation d'un bassin à partir d'un autre, avec des étapes longues et des effets fondateurs puissants, et non seulement à une adaptation aux conditions écologiques.

\section{BIBLIOGRAPHIE}

DAGNELIE P., 1982. Analyse statistique à plusieurs variables. Presse agronomique, Gembloux, Belgique, $362 \mathrm{p}$.

HEBRANT F., 1974. Problèmes de discrimination dans le cas de plusieurs populations. Biométrie, Praximétrie, 14, 3-4.

KALLEL R., 1984. La salinité des eaux de surface en Tunisie. Rapp. int. DRE, Tunisie, $55 \mathrm{p}$.

KRAIEM M.M., 1983. Les poissons d'eau douce de Tunisie. Inventaire commenté et répartition géographique. Bull. Inst. natn/ scient. techn. Océanogr. Pêche Salammbô, 10, 107-124. 
LOUDENSLAGER E. J., GALL G.A.E., 1980. Geographic patterns of protein variation and subspeciation in cutthroat trout, Salmo clarki. Syst. Zool., 29 (1), 27-42.

RAO C.R., 1953. Discriminant functions for genetic differentiation and selection. Sankhya, 12, 229-246.

RAO C.R., 1973. Recent trends of research in multivariate analysis. Biometrics, 28, 3-22.

ROUVIER R., 1966. L'analyse en composantes principales : son utilisation en génétique et ses rapports avec l'analyse discriminante. Biometrics, 22, 334-357.

SNEATH P.H.A., SOKAL R.R., 1973. Numerical taxonomy. The principles and practice of numerical classification. FREEMAN, San Francisco, $573 \mathrm{p}$.

YOCCOZ N.G., 1988. Le rôle du modèle euclidien d'analyse des données en biologie évolutive. Thèse Doct., Univ. Lyon I, 254 p.

ZAIEM M.H., 1988. Les méthodes exploratrices de l'analyse des données. Univ. Tunis, Inst. sup. Educ. Form. cont., 225 p. 